\title{
Correction and accuracy improvement of non-parallel shear zone model
}

\author{
Yaoke Wang ${ }^{1}$, Meng Kou ${ }^{1}$, Wei Ding ${ }^{1}$, Huan $\mathrm{Ma}^{1}$ and Liangshan Xiong ${ }^{1,2, a}$ \\ ${ }^{1}$ Huazhong University of S \& T, School of Mechanical Science and Engineering, Luoyu 1037, Wuhan, China \\ ${ }^{2}$ Huazhong University of S \& T, State Engineering Research Center of Digital Manufacturing and Equipment, Luoyu 1037, Wuhan, China
}

\begin{abstract}
When applying the non-parallel shear zone model to predict the cutting process parameters of carbon steel workpiece, it is found that there is a big error between the prediction results and the experimental values. And also, the former approach to obtain the relevant cutting parameters of the non-parallel shear zone model by applying coordinate transformation to the parallel shear zone model has a theoretical error - it erroneously regards the determinant $(|J|)$ of the Jacobian matrix $(J)$ in the coordinate transformation as a constant. The shape of the shear zone obtained when $|J|$ is not constant is drew and it is found that the two boundaries of the shear zone are two slightly curved surfaces rather than two inclined planes. Also, the error between predicted values and experimental values of cutting force and cutting thrust is slightly smaller than that of constant $|J|$. A corrected model where $|J|$ is a variable is proposed. Since the specific values of inclination of the shear zone $(\alpha, \beta)$, the thickness coefficient of the shear zone $\left(a_{s}\right)$ and the constants related to the material $\left(f_{0}, p\right)$ are not given in the former work, a method to obtain the above-mentioned five constants by solving multivariable constrained optimization problem based on experimental data was also proposed; based on the obtained experimental data of AISI 1045 steel workpiece cutting force, cutting thrust, chip thickness, the results of five abovementioned model constants are obtained. It is found that, compared with prediction from uncorrected model, the cutting force and cutting thrust of AISI 1045 steel predicted by the corrected model with the obtained constants has a better agreement with the experimental values obtained by Ivester.
\end{abstract}

\section{Introduction}

To build cutting analysis model is an effective way to quantitatively analyze different kinds of phenomena and output (cutting force, cutting temperature, chip deformation and workpiece quality) in the cutting process and has long been paid attention by many researchers [1-4].

In some sense, the development of cutting theory is to establish new, more accurate analysis model which is more consistent with the actual situation to replace the old, poorly-accurate analysis model which is relatively inconsistent with the actual situation. In order to make the cutting analysis model more accurate in predicting the cutting process, the predecessors have conducted various observations, analyses, and assumptions on the physical processes and geometric features in metal cutting. Then, they have proposed a series of new cutting analysis models accordingly.

An analysis and prediction theory based on parallel plane shear zones proposed by Oxley and his co-workers [5] provides a mathematical model suitable for the prediction of the cutting process parameters of carbon steel and aluminum alloy workpieces [6]. In this theory, the shear zone is regarded as a strip zone bounded by two parallel planes and bisected by the primary shear plane, which is more consistent with the actual situation than the Merchant's shear plane model [1]. Also, since the effect of work hardening and temperature softening on workpiece deformation is taken in to consideration, a more accurate prediction result can be obtained.

Astakhov [7,8] and Leopold [9] obtained and analyzed the metallographic photographs of the chips obtained from orthogonal cutting of stainless steel and carbon steel. It was found that the shear zone consisted of two sections of unequal thickness (the first section was wide, where the chip speed changes slowly; the second one was narrow, where the chip speed changes rapidly) rather than the symmetrical strip area bisected by the primary shear plane. Based on this finding, Tounsi etc. [10] proposed a method for solving the coefficients of constitutive equation of workpiece material based on the cutting experiment data obtained under orthogonal cutting conditions. Li $[11,12]$ further established unequal division shear-zone model which assumed that the flow velocity of the cut material in the shear zone obeyed a piecewise power-law distribution, and also, applied the thermal control equation in the shear zone to predict the cutting process parameters under orthogonal cutting conditions. The validity of this model has been verified in stainless steel orthogonal cutting experiments: the predicted cutting force and cutting thrust share consistent trends with the experimental data while feed rate, cutting speed and rake angle changes, but there was a big error between the predicted results and the results of cutting experiments (Up 
to $54 \%)$.

'By analyzing the metallography from orthogonal cutting on carbon steel and aluminum alloy workpiece and the relationship between shear strain and shear angle, Jesper [13] found that the two boundaries of shear zone (the initial shear plane and ultimate shear plane) are not parallel, and the initial shear plane and the ultimate shear plane have an inclination of about $0^{\circ}$ to $9^{\circ}$ relative to the main shear plane. On the basis of this, Zhou [14] applied the coordinate transformation method to the unequal division shear zone model, which is based on parallel shear planes, and cleverly obtained the formula of non-parallel shear zone model from a calculation formula of the unequal division shear zone model by using a set of transformation matrices. Then, the solution of the cutting force and cutting thrust of the non-parallel shear zone model is obtained. Unfortunately, although the prediction accuracy of the non-parallel shear zone model is higher than the unequal division shear zone model, the prediction results are still far from the experimental data (Error up to $28 \%$ ).

When learning and applying the solution of Zhou, it was found that, in order to simplify the process of solving, this solution artificially regarded the determinant $(|J|)$ of the Jacobian matrix $(J)$ used in calculating the coordinate value of the boundary of the transformed shear zone as a constant. Obviously, this approach greatly weakened the mathematic rigor of the proposed solution and may increase the error of the results obtained.

This paper first proves that $|J|$ used by Zhou in coordinate transformation and solution is not a constant, and its values are different at different stages of the solution process. Then, on basis of the inverse method to obtain the theoretical model coefficients based on the experimental data proposed by the predecessors [15-19], a method to obtain the coefficients in non-parallel shear zone model based on experimental values of the cutting force, cutting thrust and chip thickness are proposed. By using optimization techniques, in this paper, a set of reversely determined coefficients were obtained by a set of orthogonal cutting experimental data of cutting force, cutting thrust and chip thickness of a AISI 1045 steel workpiece. Finally, in order to verify the validation of the method and the accuracy of the results obtained, this set of inversed coefficients is applied in the corrected nonparallel shear zone model. The obtained results of cutting force and cutting thrust of AISI 1045 steel workpiece are compared with the experimental results of Ivester etc. [25]. The results show that the prediction error of the corrected model is smaller than that of Zhou [14].

\section{Defect and Correction of Non-Parallel Shear Zone Model}

\subsection{Introduction of Non-Parallel Shear Zone Model and its solving method}

Figure. 1 is a schematic diagram of non-parallel shear zone model. The calculation formula can be obtained from the unequal division shear zone model shown in Figure. 2 by establishing a certain one-to-one correspondence to relate the four boundary points $\left(\mathrm{C}^{\prime} \mathrm{D}^{\prime} \mathrm{F}^{\prime} \mathrm{E}^{\prime}\right)$ of the former and four boundary points (CDFE) of the latter.

Assume the mapping relationship between the $x-y$ coordinate values and the $\xi-\eta$ coordinate values of the boundary points is:

$$
\left\{\begin{array}{l}
x_{i}=a_{0}+a_{1} \xi_{i}+a_{2} \eta_{i}+a_{3} \xi_{i} \eta_{i} \\
y_{i}=b_{0}+b_{1} \xi_{i}+b_{2} \eta_{i}+b_{3} \xi_{i} \eta_{i}
\end{array} \quad(i=1,2,3,4)\right.
$$

From the mapping theorem, for any function (the distribution function of the shear strain rate in Zhou's model), its partial differential mapping relationship is:

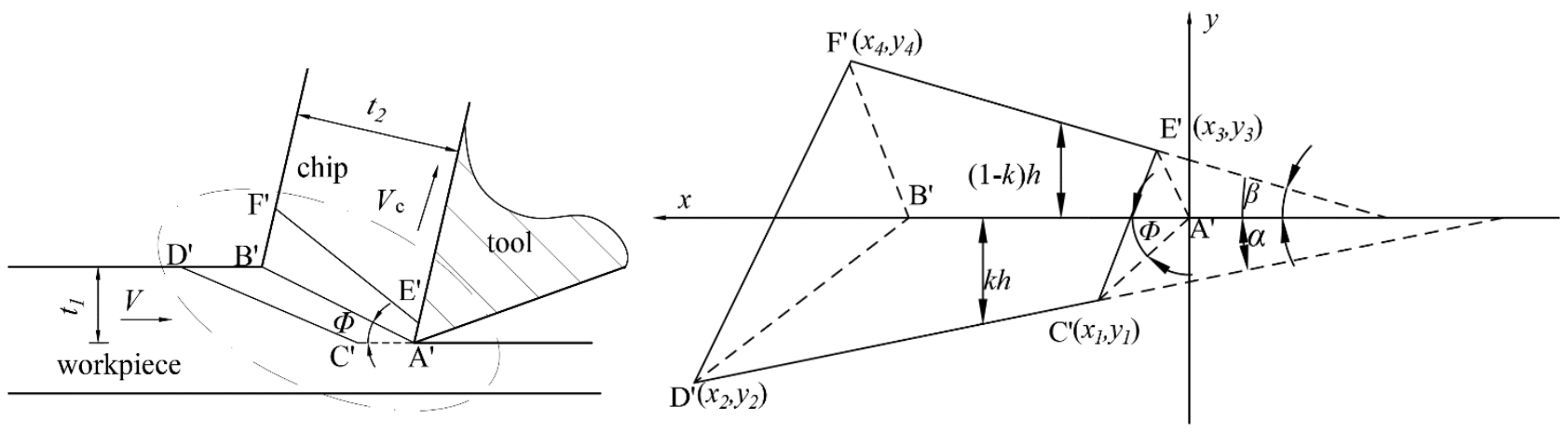

Figure 1. Non-Parallel Shear Zone Model.

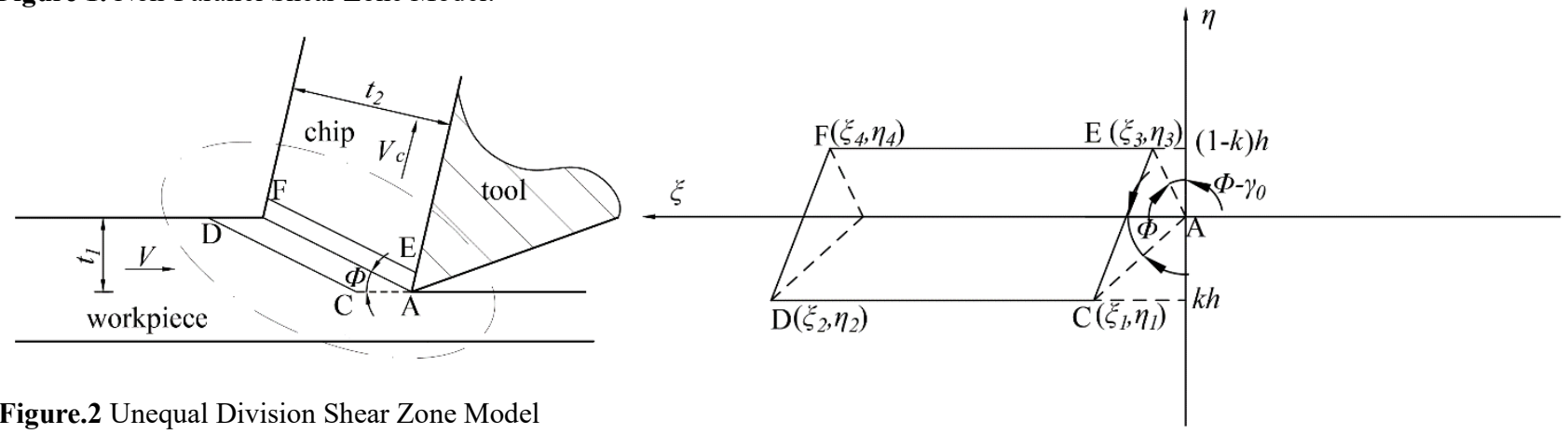




$$
\left[\begin{array}{l}
\frac{\partial \psi}{\partial x} \\
\frac{\partial \psi}{\partial y}
\end{array}\right]=J^{-1}\left[\begin{array}{l}
\frac{\partial \psi}{\partial \xi} \\
\frac{\partial \psi}{\partial \eta}
\end{array}\right], J=\left[\begin{array}{ll}
\frac{\partial x}{\partial \xi} & \frac{\partial y}{\partial \xi} \\
\frac{\partial x}{\partial \eta} & \frac{\partial y}{\partial \eta}
\end{array}\right]
$$

$J$ is the Jacobian matrix that represents the transformation process.

Through the coordinate transformation determined by equations (1) and (2), the parallel plane shear zone can be transformed to the non-parallel plane shear zone, and the calculation formula of non-parallel shear zone model can be obtained by the calculation formula applied in parallel plane shear zone.

\subsection{Proof that $|J|$ is not a Constant}

In the process of transforming the parallel plane shear zone to the non-parallel plane shear zone, in order to realize the calculation of the shear strain rate at each point in the shear zone, the value of $|J|$ needs to be calculated. In the derivation process, Zhou [14] took the value of $|J|$ at the center of gravity of the quadrilateral CDFE as the value of $|J|$ at any point in the entire shear region:

$$
|J|=1+f\left(\alpha, \beta, \Phi, \gamma_{0}, k\right)
$$

which means that $|J|$ is a constant unrelated to $\xi$ and $\eta$. $|J|$ is:

From Equations (1) (2), the original expression of

$$
|J|=a_{1} b_{2}-b_{1} a_{2}+\left(b_{2} a_{3}-b_{3} a_{2}\right) \eta+\left(b_{3} a_{1}-b_{1} a_{3}\right) \xi
$$

Then when the coefficients of $\xi$ and $\eta$ are not $0,|J|$ is a function of $\xi, \eta$, which means its value changes as the coordinate values changes. Actually, the value of $|J|$ does change with the position of the point $(\xi, \eta)$ in the shear zone (the quadrilateral CDFE ), and is not a constant. The proof is as follows.

The necessary condition for the formulas (3) and (4) is

Which equals to:

$$
\left\{\begin{array}{l}
b_{2} a_{3}-b_{3} a_{2}=0 \\
b_{3} a_{1}-b_{1} a_{3}=0
\end{array}\right.
$$

$$
\frac{b_{3}}{a_{3}}=\frac{b_{2}}{a_{2}}=\frac{b_{1}}{a_{1}}=\text { Const. }
$$

Then, that means:

$$
\frac{y_{i}-b_{0}}{x_{i}-a_{0}}=\frac{b_{1} \xi_{i}+b_{2} \eta_{i}+b_{3} \xi_{i} \eta_{i}}{a_{1} \xi_{i}+a_{2} \eta_{i}+a_{3} \xi_{i} \eta_{i}}=\text { Const. }
$$

Equation (8) is actually an equation of a line passing through $\left(a_{0}, b_{0}\right)$, which means, if the value of $|J|$ in the equation is constant, the four known points E, F, C, and D must be at the same straight line. This obviously contradicts the fact that CDFE is a quadrilateral. Thus, the value of $|J|$ should not be a constant for this coordinate transformation.

\subsection{Influence when $|J|$ is taken as a constant}

It has been calculated and verified that although the relative error of $|J|$ obtained when $|J|$ is taken as a constant is not large, only about $1 \% \%$, the model is still affected by the following three aspects.
(1) The calculation of $|J|$ is simplified. If $|J|$ is not a constant, the computational flow of the entire model can be represented by Figure 3, which is more complicated. When $|J|$ is a constant, the "point-by-point calculation" process of $|J|$ in Figure. 3 is omitted, and the integral term (proportional to the shear strain rate) in the integration process to obtain the temperature field distribution becomes conventional power functions, which makes the integration calculation process relatively simple.

(2) The distortion of shape of the transformed shear zone was caused. By further calculation, it is found that if $|J|$ is not a constant, the boundary of the non-parallel shear zone is not a plane, but an outward convex surface which is shown in Figure 4.

(3) The prediction error of the model is increased. When $|J|$ is regarded as a constant and a non-constant

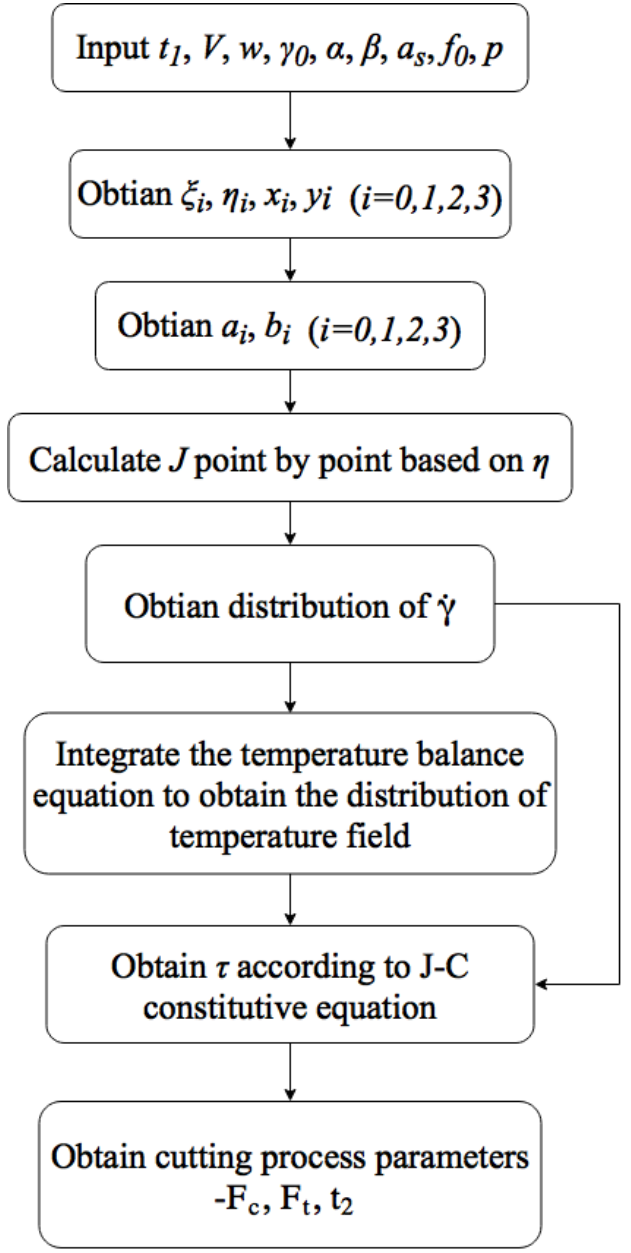

Figure.3 Computational Flow

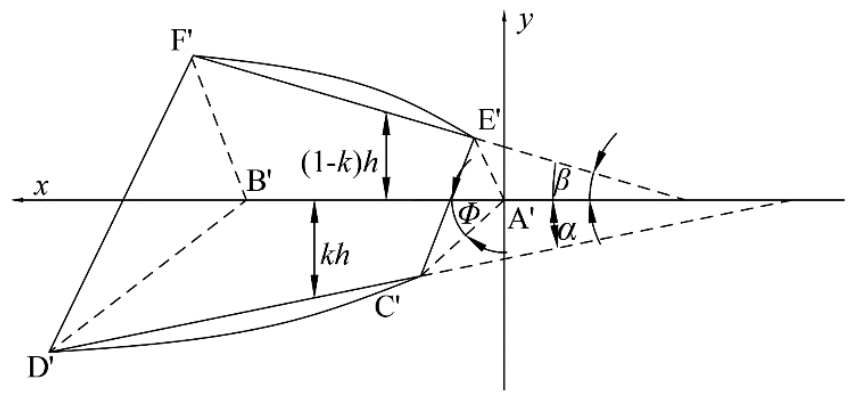

Figure.4 Actual Shear Zone Diagram 
number, the model's forecast results under a particular cutting condition are shown in Figure 5. It can be seen that, in most cases, using the constant $|J|$ actually artificially increases the prediction error of the model (For example, when the cutting conditions are $V=187.2 \mathrm{~m} / \mathrm{min}, t_{l}=0.2 \mathrm{~mm}$, $\gamma_{0}=0^{\circ}$, the prediction error of the cutting force increased by $7.6 \%$; the prediction error of the cutting thrust increased by $3.7 \%$ )

It can be seen from Figure. 5 that correcting Zhou's mistake and taking the value of $|J|$ as a non-constant number improves the prediction accuracy of the model although the forecasting error is still large.

In terms of narrative convenience, the non-parallel shear zone model where the value of $|J|$ is changed to nonconstant is called "corrected non-parallel shear zone model" and referred to as "corrected model" for short.

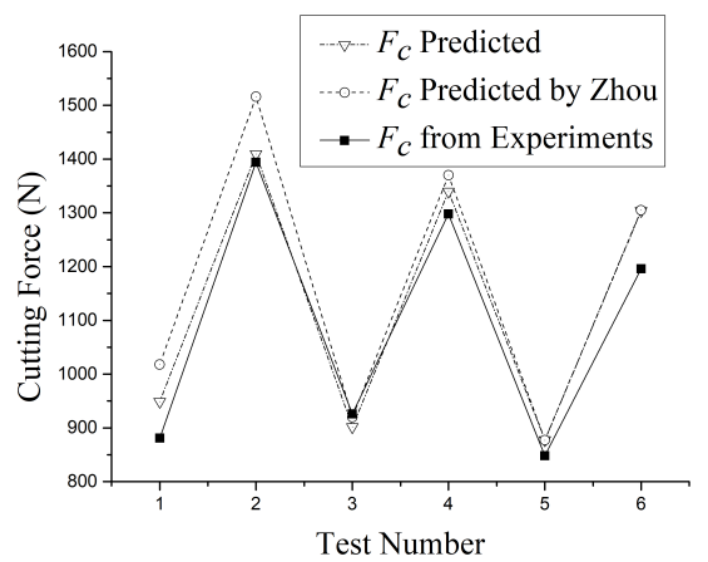

(a)

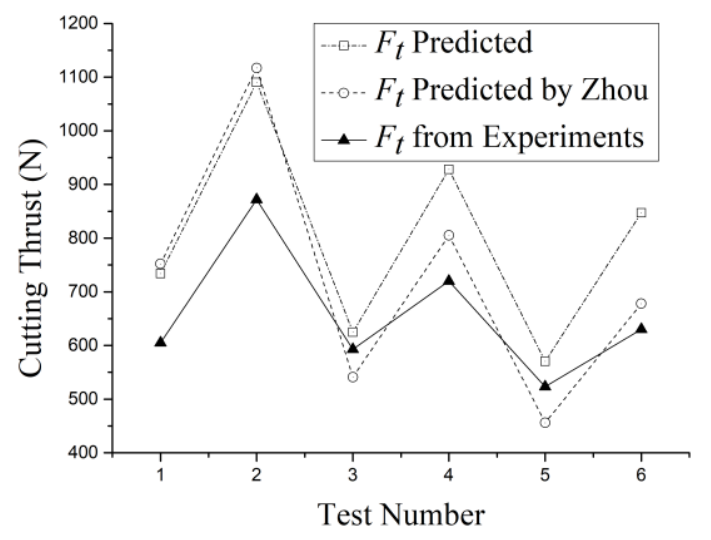

(b)

Figure.5 Predicted and Experimental Cutting Parameters (a) Comparison on Cutting Force; (b) Comparison on Cutting Thrust

\section{Coefficient and Constant Inverse of Corrected Model}

\subsection{Strategy of Coefficient and Constant Inverse}

The reason why the non-parallel shear zone model prediction accuracy is not exceptional is due to many factors, one of which is that many coefficients and constants used in the model, especially the constants defining the shape of the non-parallel shear zone, the coefficients related to the degree of chip deformation and the state of knife-chip friction $\left(\alpha, \beta, a_{s}, f_{0}, p\right)$ are all artificially given. These constants and coefficients are difficult to completely agree with the actual situation.

It is not difficult to understand that the values of the constants $\alpha, \beta, a_{s}, f_{0}, p$ should be determined by the cutting process according to its inherent laws. Only when the values of these constants meet the inherent laws of the cutting process, the output parameters of the cutting process, such as $F_{c}, F_{t}$, and $t_{2}$, are consistent with the actual situation. Conversely, if cutting force $F_{c}$, cutting thrust $F_{t}$, the ratio between cutting force and thrust $F_{c} / F_{t}$ and chip thickness $t_{2}$ predicted by the non-parallel shear zone model are consistent with the actual situation, the corresponding $\alpha, \beta, a_{s}, f_{0}, p$ is the parameter that conforms to the inherent laws of the cutting process. This provides an idea for determining the values of the constants $\alpha, \beta, a_{s}, f_{0}, p$ indirectly by measuring the force and the thickness of the chip, and further improving the prediction accuracy of the non-parallel shear zone model. The constants $\alpha, \beta, a_{s}, f_{0}, p$ which make the forecast result consistent with the experimental value are the actual values of $\alpha, \beta, a_{s}, f_{0}, p$ which are in line with the laws in metal cutting.

\subsection{Influence of Each Coefficient and Constants on Cutting Process Parameters}

It should be noted that the premise of using this idea to inversely determine the constants above is that the output parameters of the cutting process $\left(F_{c}, F_{t}, F_{c} / F_{f}\right.$ and $\left.t_{2}\right)$ which are determined by $\alpha, \beta, a_{s}, f_{0}, p$ change monotonously as $\alpha, \beta, a_{s}, f_{0}, p$ changes. So, is the actual situation not the case?

\subsubsection{Influence of Inclination $(\alpha, \beta)$ on Output Parameters of Cutting Process}

According to the study of Jaspers [13], the inclination $\alpha, \beta$ varies from 0 to $9^{\circ}$.

When the $\alpha$ and $\beta$ change from $0^{\circ}$ to $9^{\circ}$ and the other input coefficients are constant, influence on the cutting force $F_{c}$, the cutting thrust $F_{t}$, calculated by the nonparallel shear zone model is shown in Figure 6. Obviously, within a given range of $\alpha, \beta$, the cutting force $F_{c}$ and the cutting thrust $F_{t}$ are monotonous as $\alpha, \beta$ increase or decrease. In addition, from the numerical calculation, the chip thickness $t_{2}$ and the ratio of cutting force and thrust $F_{c} / F_{t}$ will not change when $\alpha, \beta$ change, which also represents a monotonous relation.

\subsubsection{Influence of the Thickness Coefficient of Shear Zone $\left(a_{s}\right)$ on Output Parameters of Cutting Process}

In order to predict the cutting force, the shear zone thickness is also one of the input conditions. Grzesik [21] proposed a relation between the thickness of the shear zone and the cutting thickness:

$$
h=\frac{t_{2}}{10 \sin \Phi}
$$




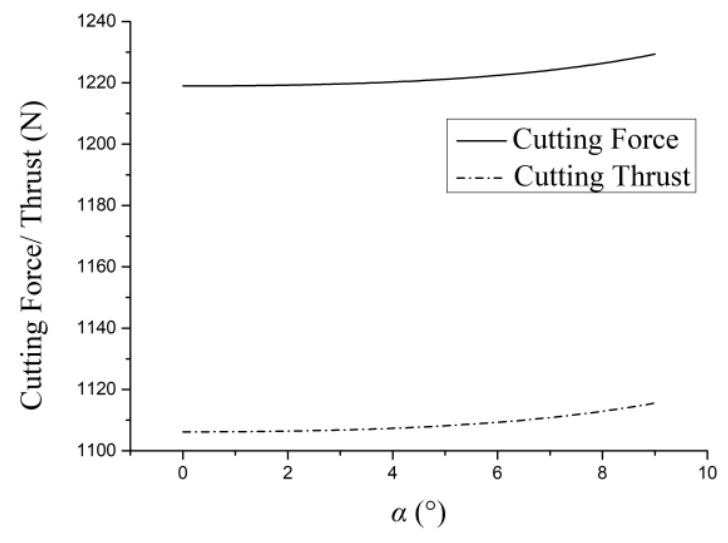

(a)

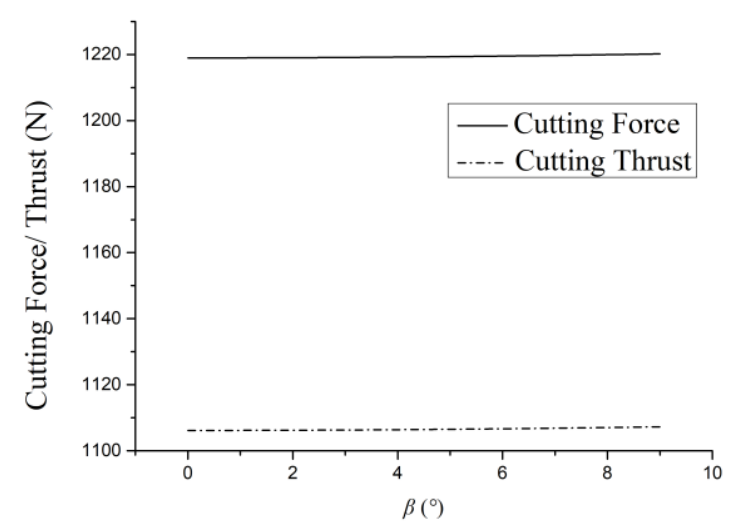

(b)

Figure. $6 F_{c}, F_{t}$ Calculated when $\alpha, \beta$ Change

(a) $\alpha$ 's Influence on Cutting Force and Cutting Thrust; (b) $\beta$ 's Influence on Cutting Force and Cutting Thrust

where $\Phi$ is the shear angle and $t_{2}$ is the cutting thickness. According to the Merchant theory and the actual situation, $\Phi$ must be taken within the interval $\left(5^{\circ}, 45^{\circ}\right)$. Assume that:

$$
h=a_{s} \times t_{2}
$$

Thus, when $\Phi \in\left(5^{\circ}, 45^{\circ}\right)$, from equation (9):

$$
a_{s} \in(0.14,1.14)
$$

To increase the reliability of the analysis process, set that $a_{s} \in(0.1,1.5)$.

When $a_{s}$ changes from 0.1 to 1.5 and other input coefficients are constant, by numerical simulation, it is found that $F_{c}, F_{t}, t_{2}, F_{c} / F_{t}$ calculated by the non-parallel shear zone model are monotonically related to $a_{s}$, which is similar to the relations with $\alpha, \beta$.

\subsubsection{Influence of the Knife-Chip Friction Coefficients $\left(f_{0}, p\right)$ on Output Parameters of Cutting Process}

The coefficients $f_{0}$ and $p$ are mainly related to the friction coefficient. Dudzinski [22] proposed that the average chip friction coefficient between the chip and knife can be expressed as a power function with respect to the chip speed $V_{c}$ :

$$
f=f_{0} V_{c}^{p}
$$

where $f_{0}$ is a constant and $p<0$. It can be seen that the friction coefficient between the chips and knife is a nonconstant number. In this equation, $f_{0}$ represents the reference friction coefficient measured at a certain cutting speed and $p$ shows the effect of cutting speed on friction.

When $f_{0}$ varies from 0.6 to $1.5, p$ varies from -0.4 to 0 , and other input coefficients are constant, by numerical simulation, it is found that the corresponding $F_{c}, F_{t}, t_{2}$, $F_{c} / F_{t}$ are all monotonically related to $f_{0}, p$,

\subsubsection{Summary of Influence of $\left(\alpha, \beta, a_{s}, f_{0}, p\right)$}

In summary, $F_{c}, F_{t}, t_{2}$ and $F_{c} / F_{t}$ all monotonically change with changes in coefficient / constants $\left(\alpha, \beta, a_{s}, f_{0}, p\right)$ which are to be determined. Assume that the functions representing these relations are respectively $f_{1}, f_{2}, f_{3}$ and $f_{4}$, with the results of numerical simulation mentioned above, it can be concluded that:

$$
\left\{\begin{array}{c}
F_{c}=f_{1}\left(+f_{0},-p,+\alpha,-a_{s},+\beta\right) \\
F_{t}=f_{2}\left(+f_{0},-p,+\alpha,-a_{s},+\beta\right) \\
\frac{F_{c}}{F_{t}}=f_{3}\left(-f_{0},+p\right) \\
t_{2}=f_{4}\left(+f_{0},-p\right)
\end{array}\right.
$$

In the equation, the positive sign before each parameter indicates that the function is positively related to the desired parameter; the negative sign indicates that the function is negatively related to the desired parameter.

\subsection{Inverse of Coefficients and Constants of the Model}

\subsubsection{Design and Data of Cutting Experiment}

Based on the strategy introduced above, modern optimization techniques are used to retrieve the above coefficients and constants from the experimental data of the cutting process parameters $F_{c}, F_{t}, F_{c} / F_{f}$, and $t_{2}$.

To obtain the forces $F_{c 0}, F_{t 0}$ and the chip thickness $t_{20}$ of the orthogonal cutting, the experiment was carried out on a Mazak CNC lathe. The tool was sharpened YT15 carbide turning tool; the workpiece material was AISI 1045 steel, the workpiece shape was a circular tube shaped from bar stock, the outer diameter was $80 \mathrm{~mm}$, and the inner diameter was determined by required cutting width $w$; the Kistler 9257B three-way dynamometer is used to measure the cutting forces $F_{c 0}$ and $F_{t 0}$, and a truncated caliper is used to measure $t_{20}$. In order to eliminate the influence of factors such as built-up edge and size effect on the test results, the cutting speed $U \geq 100 \mathrm{~m} / \mathrm{min}$ and the undeformed cutting thickness $t_{1} \geq 0.1 \mathrm{~mm}$ are guaranteed during the experiments. In order to reduce the number of experiments, the designed cutting experiment was a 4 factor 5 level orthogonal cutting experiment and a 5-6 orthogonal table is applied. In order to eliminate the error caused by accidental factors as much as possible, each cutting condition is repeated twice, and the average value of the two measured cutting forces is taken as the actual value of the cutting force. The thicknesses of the chip were measured five times at different positions where the 
cutting in and cutting out points of the tool are avoided, and the average value was taken as the measured value of the cutting thickness. Then, the average value of values measured in two cutting experiments was taken as the final measured value.

Experimental Data is shown in Table 1:

Table 1. Experimental Data

\begin{tabular}{|c|c|c|c|c|c|c|c|}
\hline & $\gamma_{0}$ & $t_{1}$ & $w$ & $\mathrm{~V}$ & $F_{\mathrm{c} 0}$ & $F_{\mathrm{t} 0}$ & $t_{20}$ \\
\hline U. & $\mathrm{N}$ & $\mathrm{mm}$ & $\mathrm{mm}$ & $\begin{array}{l}\mathrm{m} / \\
\min \end{array}$ & $\mathrm{N}$ & $\mathrm{N}$ & $M m$ \\
\hline 1 & -9.83 & 0.10 & 1.00 & 100 & 383 & 346 & 0.350 \\
\hline 2 & -9.83 & 0.15 & 1.31 & 150 & 631 & 524 & 0.420 \\
\hline 3 & -9.83 & 0.20 & 1.50 & 200 & 877 & 648 & 0.490 \\
\hline 4 & -9.83 & 0.25 & 1.81 & 250 & 1140 & 761 & 0.560 \\
\hline 5 & -9.83 & 0.30 & 1.90 & 300 & 1462 & 909 & 0.630 \\
\hline 6 & -4.67 & 0.10 & 1.31 & 200 & 446 & 417 & 0.280 \\
\hline 7 & -4.67 & 0.15 & 1.50 & 250 & 707 & 595 & 0.370 \\
\hline 8 & -4.67 & 0.20 & 1.81 & 300 & 94 & 685 & 0.470 \\
\hline 9 & -4.67 & 0.25 & 1.90 & 100 & 1354 & 915 & 0.630 \\
\hline 10 & -4.67 & 0.30 & 1.00 & 150 & 80 & 488 & 0.440 \\
\hline 11 & -0.50 & 0.10 & 1.50 & 300 & 477 & 416 & 0.250 \\
\hline 12 & -0.50 & 0.15 & 1.81 & 100 & 779 & 578 & 0.370 \\
\hline 13 & -0.50 & 0.20 & 1.90 & 150 & 1023 & 664 & 0.470 \\
\hline 14 & -0.50 & 0.25 & 1.00 & 200 & 591 & 315 & 0.550 \\
\hline 15 & -0.50 & 0.30 & 1.31 & 250 & 839 & 410 & 0.640 \\
\hline 16 & 4.00 & 0.10 & 1.81 & 150 & 522 & 401 & 0.285 \\
\hline 17 & 4.00 & 0.15 & 1.90 & 200 & 754 & 524 & 0.357 \\
\hline 18 & 4.00 & 0.20 & 1.00 & 250 & 469 & 280 & 0.423 \\
\hline 19 & 4.00 & 0.25 & 1.31 & 300 & 676 & 366 & 0.532 \\
\hline 20 & 4.00 & 0.30 & 1.50 & 100 & 1037 & 542 & 0.669 \\
\hline 21 & 10.67 & 0.10 & 1.90 & 250 & 487 & 313 & 0.236 \\
\hline 22 & 10.67 & 0.15 & 1.00 & 300 & 332 & 185 & 0.303 \\
\hline 23 & 10.67 & 0.20 & 1.31 & 100 & 555 & 264 & 0.455 \\
\hline 24 & 10.67 & 0.25 & 1.50 & 150 & 798 & 356 & 0.536 \\
\hline 25 & 10.67 & 0.30 & 1.81 & 200 & 1013 & 392 & 0.615 \\
\hline 26 & -6.50 & 0.10 & 1.00 & 100 & 343 & 295 & 0.306 \\
\hline 27 & -6.50 & 0.15 & 1.31 & 150 & 593 & 451 & 0.401 \\
\hline 28 & -6.50 & 0.20 & 1.50 & 200 & 827 & 572 & 0.486 \\
\hline 29 & -6.50 & 0.25 & 1.81 & 250 & 1164 & 755 & 0.563 \\
\hline 30 & -6.50 & 0.30 & 1.90 & 300 & 1383 & 840 & 0.636 \\
\hline 31 & 3.17 & 0.10 & 1.31 & 200 & 373 & 273 & 0.258 \\
\hline 32 & 3.17 & 0.15 & 1.50 & 250 & 570 & 364 & 0.346 \\
\hline 33 & 3.17 & 0.20 & 1.81 & 300 & 845 & 495 & 0.425 \\
\hline 34 & 3.17 & 0.25 & 1.90 & 100 & 1142 & 635 & 0.587 \\
\hline 35 & 3.17 & 0.30 & 1.00 & 150 & 661 & 315 & 0.643 \\
\hline 36 & 6.50 & 0.10 & 1.50 & 300 & 395 & 275 & 0.238 \\
\hline
\end{tabular}

\begin{tabular}{l|cccc|ccc}
\hline 37 & 6.50 & 0.15 & 1.81 & 100 & 706 & 443 & 0.380 \\
38 & 6.50 & 0.20 & 1.90 & 150 & 893 & 502 & 0.453 \\
39 & 6.50 & 0.25 & 1.00 & 200 & 538 & 255 & 0.521 \\
40 & 6.50 & 0.30 & 1.31 & 250 & 798 & 364 & 0.585 \\
41 & 8.83 & 0.10 & 1.81 & 150 & 488 & 337 & 0.256 \\
42 & 8.83 & 0.15 & 1.90 & 200 & 680 & 405 & 0.34 \\
43 & 8.83 & 0.20 & 1.00 & 250 & 432 & 212 & 0.415 \\
44 & 8.83 & 0.25 & 1.31 & 300 & 663 & 309 & 0.484 \\
45 & 8.83 & 0.30 & 1.50 & 100 & 950 & 431 & 0.647 \\
46 & 14.00 & 0.10 & 1.90 & 250 & 458 & 286 & 0.230 \\
47 & 14.00 & 0.15 & 1.00 & 300 & 319 & 158 & 0.309 \\
48 & 14.00 & 0.20 & 1.31 & 100 & 567 & 267 & 0.449 \\
49 & 14.00 & 0.25 & 1.50 & 155 & 744 & 325 & 0.506 \\
50 & 14.00 & 0.30 & 1.81 & 200 & 1013 & 421 & 0.566 \\
\hline
\end{tabular}

\subsubsection{Objective Function and Constraint function}

According to the strategy above, the values of $\alpha, \beta, a_{s}, f_{0}$, $p$ that make the prediction result closest to the experimental data are the values that match the cutting rule best. Then, the values of $\alpha, \beta, a_{s}, f_{0}, p$, which is the closest to the predicted cutting process parameters and the experimental results as a whole are the demanded values.

Construct the objective function:

$$
\begin{aligned}
& \min _{x} F(x)=\sum_{i=1}^{n}\left[\left(\frac{t_{2 i}}{t_{20 i}}-1\right)^{2}+\right.\left.\left(\frac{F_{t i}}{F_{t 0 i}}-1\right)^{2}+\left(\frac{F_{c i}}{F_{c 0 i}}-1\right)^{2}+\left(\frac{F_{c i}}{F_{t i}} / \frac{F_{c 0 i}}{F_{t 0 i}}-1\right)^{2}\right] \\
& \text { s.t. }\left\{\begin{array}{l}
0.25 t_{1 i}-h_{i}<0 \\
h i-t_{1 i}<0 \\
0.6-f<0 \\
f-1.5<0
\end{array}\right.
\end{aligned}
$$

In the objective function above, $x$ is the variable to be optimized $\left(x=\left[\alpha, \beta, a_{s}, f_{0}, p\right]\right) ; t_{20}$ is the experimentally measured chip thickness; $t_{2}$ is the predicted chip thickness; $F_{c 0}$ is the experimentally measured cutting force; $F_{c}$ is the predicted cutting force; $F_{t 0}$ is the experimentally measured cutting thrust; $F_{t}$ is the cutting thrust; $h$ is the thickness of the shear zone; $t_{l}$ is the cutting thickness; $f$ is the average friction coefficient between the knife and chips.

\subsubsection{Optimization Algorithm}

The experimental data can be used to optimize the objective function above using the optimization functions $\boldsymbol{g} \boldsymbol{a}$ and fmincon provided by the Matlab2014a software platform to obtain the desired model coefficients and constants $\alpha, \beta, a_{s}, f_{0}, p$.

Specific Process :

(1) First use the genetic algorithm $\boldsymbol{g} \boldsymbol{a}$ to search in a larger range to get a rough solution $x_{0}$;

(2) Set $x_{0}$ as the center and rebuild a smaller search interval. If the original interval width is $B$, the upper limit of the original interval is $U B$ and the lower limit is $L B$, then the new interval is $\left[\max \left(X_{0}-0.5 a B, L B\right), \min \left(X_{0}-0.5 a B, U B\right)\right]$ (the coefficient $a$ ranges from 0 to $1(0<a<1)$;

(3) Taking $x_{0}$ as the initial value and also taking the new 
interval as the range, the fmincon function is used to obtain a smaller objective function value in the new search interval. The corresponding solution $x_{1}$ is the demanded solution.

\subsubsection{The result of the solution}

Through the process above, according to the experimental data in Table 1, the following optimization results can be obtained:

$$
\alpha=0.9596^{\circ} ; \beta=0.6142^{\circ} ; a_{s}=1 ; f_{0}=0.703 ; p=-0.175
$$

For convenience of narrative, coefficients obtained above is referred to as optimized coefficients.

\section{Prediction Accuracy of Revised Model with Optimized Coefficients}

\subsection{Experimental Data for Comparison}

To verify the validity of the proposed method, the two boundaries of the cutting forces' and cutting thrusts' reference values based on four groups of AISI 1045 steel cutting processes which were measured by R. W. Ivester etc [19] are used. The cutting forces and cutting thrusts were calculated for AISI 1045 steel orthogonal cutting under the exact cutting conditions.

\subsection{Prediction Accuracy of Revised Model With Optimized Coefficients}

The prediction results of cutting process parameters of Zhou's model and revised model after coefficients/ constants re-determination are shown in Figure 7. As can be seen from the figures, most of the prediction accuracy after correction is higher than that of Zhou's. Only a few data have lower prediction accuracy than Zhou's after eliminating several groups of data that neither models can predict preferably. After calculation, the cutting forces' average prediction accuracy predicted by revised model is only increased by $0.4 \%$, but the cutting thrust forces' prediction accuracy is improved from $22 \%$ to $18 \%$. Hence, it can be concluded that the overall prediction accuracy of the corrected non-parallel shear zone model is higher than Zhou's prediction accuracy, the predicted forces are closer to experimental data.

\section{Conclusions}

The determinant of Jacobian matrix in Zhou's paper is not a constant value, conversely, it should be a variable.

Setting $|J|$ as constant value could result in the deformation of shear region in non-parallel shear region model and the increasement of cutting force's prediction error.

Revised model to solve the above problem is proposed.

A method using modern optimization methods to reversely calculate the five coefficients $\left(\alpha, \beta, a_{s}, f_{0}, p\right)$ with experimental values (cutting force, cutting thrust and chip thickness) in corrected model is proposed.

Designed and finished a set of cutting experiments based on AISI 1045 Steel workpieces orthogonal cutting to obtain cutting force, cutting thrust and chip thickness.

According to the methods and experimental data above, the specific values of five optimized coefficients are obtained while applied revised model in AISI 1045 steel workpieces; The average cutting thrust error is decreased from $22 \%$ of the erroneous models proposed by predecessor to $18 \%$ after applying optimized coefficients in corrected model.

\section{Acknowledges}

This research work was supported by the National Natural Science Foundation of China (No: 51675203) and the Undergraduate Research Opportunity Program of Huazhong University of Science and Technology (No: 20160205Z). The relevant cutting experiments were completed using the machine tools and test instruments of the State Key Laboratory of Digital Manufacturing Equipment \& Technology.

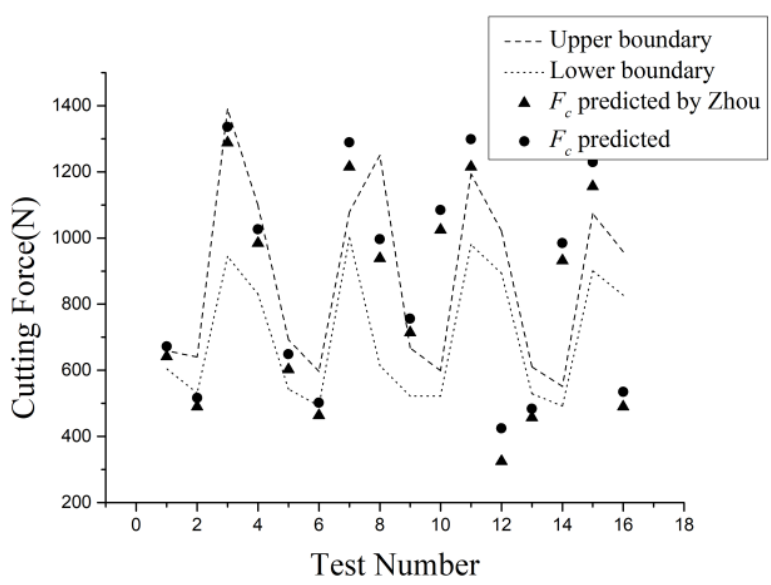

(b)

Figure.7 Cutting Process Parameters Predicted by Zhou's Model and revised model with optimized coefficients. (a) Comparison on Cutting Thrust; (b) Comparison on Cutting Force; 


\section{References}

1. H. Ernst, M.E. Merchant, T. Am. Soc. Metal., ASM 29, 299-378 (1941)

2. E.H. Lee, B.W. Shaffer, J. Appl. Mech., 18, 405-413 (1951)

3. S. Kobayashi, E.G. Thomsen, J. Eeg. Ind., 84, 71-80, (1962)

4. E.J.A. Armarego, Mach. Sci. Technol., 2(2), 191-211 (1998)

5. P.L.B Oxley, Mechanics of Machining, Chichester: Ellis Horwood (1989)

6. M.R.V. Sereshk, P. Heydarizadeh, The Evaluation of Analytical Models for Orthogonal Cutting Process, International Conference on Electrical, Automation and Mechanical Engineering (2015)

7. V.P. Astakhov, M.O.M. Osman, M.T. Hayajneh, Int. J. Mach. Tool Manu., 41(3), 393-418(2001)

8. V.P. Astakhov, Metal Cutting Mechanics, Boca Raton: CRC Press, 1998.

9. J. Leopold, Mechanical and physical models of machining, Proceedings of the Second CIRP Wordshop on Modeling of Machining Operation (1999)

10. N. Tounsi, J. Vincenti, A. Otho, et al, Int. J. Mach. Tool Manu., 42(12), 1373-1383 (2002)

11. B. Li, X. Wang, Y. Hu, et al, Int. J. Adv. Manuf. Tech., 54(5-8), 431-443 (2011)

12. B. Li, Y. Hu, X. Wang, et al., Mach. Sci. Technol., 15(4), 453-484 (2011)

13. S.P.F.C. Jaspers, J.H. Dautzenberg, J. Mater. Process Tech., 121, 123-135 (2002)

14. F. Zhou, X. Wang, Y. Hu, J. Appl. Mech. Mater., 229, 503-506 (2012)

15. T. Dereli, I. H. Filiz, A. Baykasoglu, Int. J. Prod. Res., 39(15), 3303-3328 (2001)

16. P. Asokan, R. Saravanan, K. Vijayakumar, Int. J. Adv. Manuf. Tech., 21(1),1-9 (2003)

17. P.E. Amiolemhen, A.O. A Ibhadode, Int. J. Mach. Tool. Мапи., 44(12-13), 1403-1412 (2004)

18. R.Q. Sardiñas, M.R. Santana, Eng. Appl. Artif. Intel., 19(2), 127-133 (2006)

19. L. Xiong, J. Wang, Y. Gan, et al., Int. J. Adv. Manuf. Tech., 77(1-4), 1-13 (2015)

20. R.W. Ivester, M. Kennedy, M. Davies et al., Mach. Sci. Technol., 4(3), 511-538 (2000)

21. W. Grzesek, Advanced Machining Processes of Metallic Materials, London: Elsvier (2008)

22. D. Dudzinski, A. Molinari, Int. J. Mech. Sci., 39(4), 369-389 (1997) 